\title{
Estudio, restauración y musealización: teoría y práctica. La Colección de Pintura La Canal-Blaya
}

\author{
Germán Ramallo Asensio \\ Universidad de Murcia

\begin{abstract}
RESUMEN: La Colección fue cedida por sus propietarios a la parroquia de San Miguel, de Mula (Murcia, España) en 1943 y, desde entonces, no se había intervenido en ella. La labor de catalogación y restauración se hizo en cuatro cursos de verano, de 1997 a 2001. En ellos se combinó la docencia teórica con la práctica que se aplicó a la restauración de las pinturas. Se encargó de ello un cualificado profesorado de Historia del Arte e importantes restauradores de los mejores museos españoles. Al finalizar el proceso, todo lo estudiado y restaurado pasó a exhibirse en el Museo Parroquial. La Colección es importante por reunir cuadros de calidad del siglo XVI al XIX, alguno firmado, y también, por la gran variedad de géneros, temática, técnicas y soportes.
\end{abstract}

PALABRAS CLAVE: Pintura, Colección, Restauración, Museo, La Canal-Blaya, Mula, Murcia.

\section{Research, Restoration and Musealization: Theory and Practice. The Painting Collection of La Canal-Blaya}

\begin{abstract}
The Collection was donated by their owners to the parish of San Miguel, of Mula (Murcia, Spain) in 1943. Since then, no intervention has been done in these works. The cataloging and restoration works were done during four summer courses from 1997 to 2001. For their restoration, theoretical and practical teaching was combined. This was undertaken by highly qualified faculty members of the Art History departments from several universities of Spain, and restorers of the best Spanish museums. At the end of the process, the restored works were exhibited in the «Museo Parroquial» (San Miguel, Mula, Murcia). The collection is important because it gathers quality paintings from sixteenth to the nineteenth century (some of them signed) and also because of its wide variety of genres, themes, techniques and supports.
\end{abstract}

KEY WORDS: Painting, Collection, Restoration, Museum, The Canal-Blaya, Mula, Murcia.

Recibido: 26 de abril de 2013 / Aceptado: 20 de mayo de 2013.

\section{Las colecciones de pintura de ámbito privado}

La mayor parte del patrimonio pictórico español está ahora centrado en los museos de la más variada gestión, temática o titularidad, así como en los templos y los pocos edificios que componen el patrimonio nacional. No obstante es sabido que hay también un buen número de obras en colecciones particulares de acceso más privado, o inaccesibles sin permisos y que, en muchas ocasiones,

\footnotetext{
* RAMALlo ASENSIO, Germán: «Estudio, restauración y musealización: Teoría y práctica. La Colección de Pintura La Canal-Blaya", Boletín de Arte, n. ${ }^{\circ} 34$, Departamento de Historia del Arte, Universidad de Málaga, 2013, pp. 223-245, ISSN: 0211-8483.
} 
suponen tesoros de gran interés por guardarse en ellas auténticos hitos de la pintura o ser importantes por su carácter monográfico o de cualquier otra índole. Estas colecciones se han ido formando a lo largo del tiempo; algunas desde los inicios de la Edad Moderna y con mas frecuencia desde el siglo XVII ${ }^{1}$ y todo el siglo XVIII, momento en que era una auténtica moda entre la nobleza y clase adinerada en general, la posesión de obras de arte, emulando con ello la actitud de los más selectos y educados, incluidos en ello los mismos reyes de España y sus inmediatos cortesanos. En algunos había una auténtica fiebre por la adquisición de obras de valor o de pintores reconocidos e incluso, cuando esto no se podía conseguir, una buena copia de esas codiciadas obras maestras podía ser igualmente valorada. Se aprovechaban los viajes al extranjero para hacerse con obra de otros países aunque fuera de pequeño tamaño; muchas veces se trataba de obras falsas que se hacían pasar por auténticas o, si el comprador no era demasiado exigente, de obras de clara tercera fila.

Pero lo cierto es que había muy pocos hogares que se preciasen de medianamente cultos e ilustres que no tuvieran al menos unas cuantas decenas de cuadros y no solo de tema religioso, sino asimismo de asunto profano entre los que se podían encontrar: paisajes, cuadros de batalla, bodegones y floreros, alegoría de los continentes o estaciones del año, y otros variados temas.

A partir de los últimos años del siglo XVIII y durante buena parte del XIX cambiaron los gustos hacia cuadros de menor tamaño, pues los cuartos de las casonas y palacios también se reducían en busca de la comodidad. Asimismo aumentó también la demanda de temática profana y asuntos de género e igualmente se popularizó el retrato. Al mismo tiempo las colecciones se vieron favorecidas por la pintura religiosa que procedía de las actuaciones desamortizadoras llevadas a cabo a partir del segundo tercio del siglo XIX. Pero estos coleccionistas decimonónicos también gustaron de atesorar otras rarezas y, muy principalmente, objetos extraídos de las numerosas excavaciones que, muchas sin método, se hacían por doquier en busca del esclarecimiento del más remoto pasado. Algunas de estas colecciones han dado lugar a magníficos museos como es el caso del Lázaro Galdeano o el Cerralbo, ambos en Madrid, o el Frederic Marés, en Barcelona, pero otras muchas han pasado a engrosar los museos provinciales o locales, siendo a menudo el principal núcleo de sus fondos.

1 El panorama que se dio en la Murcia del siglo XVII está reflejado de manera muy completa en el importante estudio de AGÜERA ROS, José Carlos, Pintura y Sociedad en el siglo XVII. Murcia, un centro del barroco español, Murcia, Real Academia de Alfonso X el Sabio, 1994, pp. 341-390. 
En Murcia se reunieron también importantes colecciones de pintura y objetos de arte. Unas veces eran de formación más antigua, como por ejemplo la que iniciara don Juan de Guevara García de Alcaraz, seguramente con unas primeras aportaciones de su esposa de ilustre estirpe, doña Isabel Pérez de Meca Ponce de León que más tarde, sus descendientes, de también elevado nivel cultural, irían enriqueciendo, hasta llegar a la cantidad y cualidad de la que, todavía al día de hoy se conserva en su palacio de Lorca. Otras se fueron formando más recientemente y en poco tiempo como lo fue la de don José María D’Estoup, reunida a mediados del siglo XIX. La primera es suficientemente conocida desde antiguo y gracias a los varios artículos del investigador lorquino don Juan Guirao García, además de al completo estudio monográfico que el Dr. Muñoz Clares hizo de uno de los principales artistas que la componen: Camacho Felices ${ }^{2}$, así como otras aportaciones que han hecho que muchos de sus cuadros por la calidad de sus autores (José y Federico de Madrazo) pasen a ser considerados en obras generales de historia del arte. En cuanto a la segunda, la D’Estoup, también fue estudiada y dada a conocer como tal colección por el Dr. Martínez Ripoll que catalogó hasta 155 obras y en la actualidad se conserva en la Universidad de Murcia, institución a la que fue cedida ${ }^{3}$.

Como he dicho, otras varias habrá que aún permanecen bajo buena custodia de sus propietarios y otras más que se han ido reuniendo en tiempos más recientes. De ellas no tenemos más noticias que aquellas que proporciona el prestamo aislado de alguna obra para cualquiera de las exposiciones que ahora tanto proliferan y su procedencia y ubicación quedan designadas con el genérico "colección particular».

\section{Colección La Canal-Blaya}

Pero de todas formas y siempre solo referido al ámbito murciano, a las dos que primero hemos reseñado aún hemos de añadir una tercera que también ha pasado a ser colección pública, si bien su estudio, restauración y musealización aún se encuentra en proceso. De ella es de la que vamos a tratar en estas breves páginas, por ser en ella donde se ha actuado últimamente con criterios modernos de

2 MUÑOZ CLARES, Manuel, El pintor Pedro Camacho Felices de Alisen 1644-1716 y su entorno artístico, Murcia, Academia Alfonso X el Sabio y Ayuntamiento de Lorca, 1987.

3 MARTIÍNEZ RIPOLL, Antonio, Catálogo de las pinturas de la antigua Colección D’Estoup, de Murcia, Murcia, Academia Alfonso X el Sabio y Universidad de Murcia, 1981. 
catalogación y conservación, con vistas a que cumpla el cometido de educación ciudadana para el que sus últimos propietarios hicieron la donación.

Se trata de la conocida como Colección de la Canal-Blaya, compuesta de alrededor de ciento cincuenta pinturas, además de otro medio centenar de piezas de otras técnicas y formatos que fue donada por doña Pilar de la Canal Rosique, cumpliendo la voluntad de su ya difunto esposo don Pedro Luis Blaya y Saavedra, a la parroquia de San Miguel, de Mula y allí se encuentra [1]. El documento de donación se conserva en el archivo de la Parroquia; fue suscrito en Mula, el día 15 de marzo de 1943; está firmado por Pilar de la Canal, viuda de Blaya y el párroco de San Miguel, Esteban Monreal. Doña Pilar, según se recoge por escrito, quería cumplir el deseo de su difunto marido, que ella compartía, de fundar un Museo Parroquial y "dar esplendor a la casa de Dios de quien todo lo recibimos» ${ }^{4}$.

La colección se conservaba repartida entre Murcia y Mula y fueron sus últimos propietarios don Pedro Luis Blaya y Saavedra y doña Pilar de la Canal Rosique quienes la reunieron en Mula. La casa de Murcia era la conocida como Casa de la Cruz y estaba situada en el centro histórico de la ciudad, a la bajada del Puente Viejo. Su fachada principal se abría a la calle de Frenería y era un antiguo caserón de cronología indeterminada y dimensiones bastante monumentales, aunque muy desornamentado, a menos en su parte trasera que es la que hemos podido conocer por reproducción fotográfica antigua. Lamentablemente fue demolida a principios de la década de los sesenta del pasado siglo, como tantas otras construcciones de interés, para abrir la Gran Vía, una ancha y recta arteria que atravesando el centro de la ciudad, facilitara la circulación rodada de norte a sur. La otra casa, la de Mula, aún se conserva: ocupa el número 7 de la calle de González y es un precioso ejemplo de arquitectura señorial barroca (tiene claros elementos que la vinculan a principios del siglo XVIII) de las que tanto abundan en la localidad; aún ahora se conoce como Casa del Santo, haciendo referencia este nombre a las cualidades cristianas que según la tradición oral conservada en el pueblo adornaban a sus propietarios, tíos de don Pedro Luis, don Eulogio Saavedra y Pérez de Meca y doña María de la Concepción Blaya y Cueto, matrimonio sin hijos que, por tanto, dejaron heredero de todos sus bienes a su sobrino el citado Pedro Luis que, también por su vida devota, mantuvo el mismo apodo que había definido a su tío.

4 Recogido del documento de donación que se conserva en el Archivo de la Parroquia de Mula, suscrito en Mula a 15 de marzo de 1943 y firmado por Pilar de la Canal, viuda de Blaya y el párroco de San Miguel, Esteban Monreal. 


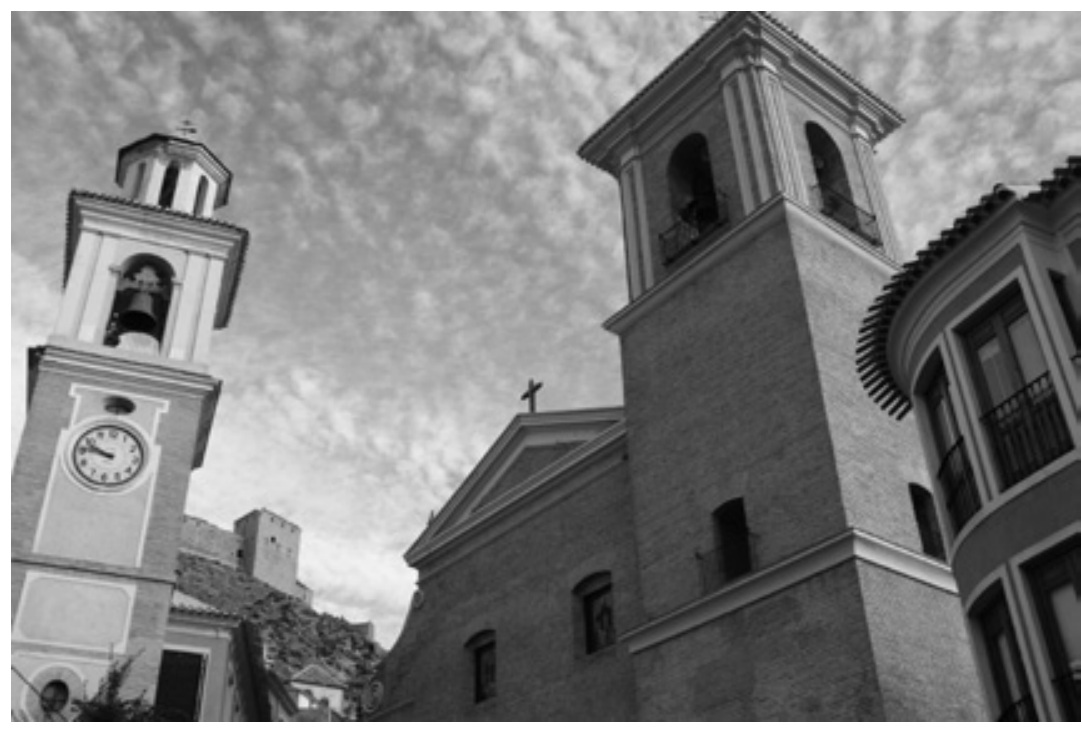

1. Mula (Murcia). Iglesia de San Miguel Arcángel. Torre del Reloj y Castillo de los Vélez

La selección y un primer inventario fueron hechos por don Manuel Muñoz Barberán, pintor y amigo de la familia donante ${ }^{5}$. Según el mismo pintor dejó escrito, la historia sucedió como sigue: «Me dijo un día “Manuel quiero ceder a la iglesia de San Miguel de Mula, algunos de estos cuadros, los mejores. Mi marido los heredó de su tío don Eulogio Saavedra Pérez de Meca con todos estos libros y los grabados. Creo que todo procede de Musso Valiente... ya usted sabe... Ayúdeme a elegir los más discretos y, por favor, haga una lista de ellos y su posible atribución ${ }^{6}$. Estas palabras son de fundamental importancia pues nos arrojan bastante luz sobre la procedencia de la colección. Según la misma doña Pilar, la colección la heredó su marido, de su tío por parte materna, don Eulogio Saavedra Pérez de Meca y a su vez este de don José Musso Valiente ${ }^{7}$. Por lo tanto, gran

5 Si bien para esa fecha era aún muy joven, Muñoz Barberán (1921-2007) llegó a alcanzar un notable reconocimiento en Murcia y aun a nivel nacional por la abundancia y calidad de obra. Realizó la decoración mural de varios templos de la región, así como la de alguno de sus teatros (Lorca y Yecla). Fue muy admirado y valorado en sus paisajes urbanos y su interpretación de temas del folclore murciano. Recibió buen número de premios y distinciones nacionales y locales. Fue asimismo erudito escritor.

6 Página preliminar al Catálogo de la Exposición, Pinturas de los siglos XVI al XIX en la colección de La Canal Blaya, de la iglesia de San Miguel de Mula, IBORRA BOTIA y MUÑOZ CLARES, Mula, 1986.

7 José Musso Valiente (1785-1837) fue un importante personaje lorquino, destacado en el panorama político e intelectual nacional y fecundo escritor. Su cualidad intelectual le fue reconocida por su ingreso en la Academia de la Historia en 1825, en la Real Academia Española en el 27 y por fin, en el 30, en la de Bellas Artes de San Fernando, siendo además en sus últimos años, bibliotecario del Ateneo de Madrid. 
parte de la colección se reuniría en Madrid y eso puede explicar su singularidad y el indudable interés artístico de algunos de sus cuadros ${ }^{8}$.

Una vez recibida la colección por la parroquia, parte de los cuadros, los de mayor tamaño y temática religiosa, fueron colgados en los muros del templo; otra parte, lo laico, se ubicó en una pequeña habitación dependencia auxiliar de la iglesia que llegó a llamarse pomposamente "Museo" y una tercera, compuesta por los objetos más pequeños, fue guardada en la casa parroquial. Así se conservó durante muchos años hasta que en 1986 se mostró de manera parcial, en una exposición organizada por la Caja de Ahorros de Alicante y Murcia, para lo que se editó un pequeño folleto en que se recogía pormenorizadamente lo expuesto, señalando título, medidas y atribución, e ilustrando con alguna fotografía en blanco y negro. Se completaba la publicación con una página preliminar redactada por don Manuel Muñoz Barberán y un breve estudio general realizado por Amparo Iborra Botía y Manuel Muñoz Clares.

Después de esto volvió todo a estar como estaba: algunos cuadros de formato grande y temática religiosa colgados en las paredes de la iglesia y los otros, guardados en dependencias de la parroquia y casa sacerdotal en condiciones no demasiado cuidadosas y por ello, acelerándose su estado de deterioro que, en algunos casos, llegó a ser verdaderamente alarmante.

Así estuvo diez años más, hasta que hacia mediados de la década de los noventa, se dieron una serie de condiciones favorables para la colección que pudieron ser aprovechadas para su proceso de catalogación, estudio y restauración. Con la llegada de un nuevo párroco a San Miguel, don Ramón García Gómez, se reavivó el interés por las obras de arte que poseía la parroquia y se

Una vida no muy larga, pero muy intensa y en relación con todos los campos de la cultura que justifica el afán coleccionista de obras de arte e incluso le haría fácil el acceso a ellas. Su relación con la ciudad natal y sobre todo su estancia en Madrid como hombre cualificado de la cultura, pueden explicar la variedad y a veces rareza, a la par que la calidad, de la pintura que llegó a reunir, difícil de conseguir en un panorama estrictamente local. Esta, como colección, hubo de llegar definitivamente a Lorca con el establecimiento en esta ciudad de su hijo don José Musso y Fontes (1812-1886) que fue concejal y diputado provincial y, también durante algún tiempo, comisario provincial de Agricultura, tema este del que tratan prácticamente todos sus numerosos escritos. CAMPOY, José María, Biografía de D. José Musso Valiente, Biblioteca El Diario de Avisos, Lorca, 1887. Ver también, AA.VV., José Musso Valiente 1785-1837. Vida y obra, Murcia, Fundación Centro de Estudios Históricos e Investigaciones Locales de la Región de Murcia, 1998. Dentro de esta obra de conjunto se estudia su faceta erudita por VILAR, J. B., "José Musso y la cultura española en la transición al liberalismo...", pp. 45-63.

8 SÁNCHEZ MAURANDI, A., Eulogio Saavedra, Imprenta Victoria, Mula, 1953. GONZÁLEZ CASTAÑO, J., "Notas sobre la vida y obra de Eulogio Saavedra Pérez de Meca», prólogo a edición del libro de Eulogio Saavedra y Pérez de Meca, Mastia y Tarteso y los pueblos litorales del Sud-Este de España en la Antigüedad, Murcia, Real Academia de Alfonso X el Sabio, Biblioteca Murciana de Bolsillo, 145, 2004, pp. 7-15.

9 Véase nota 6. 


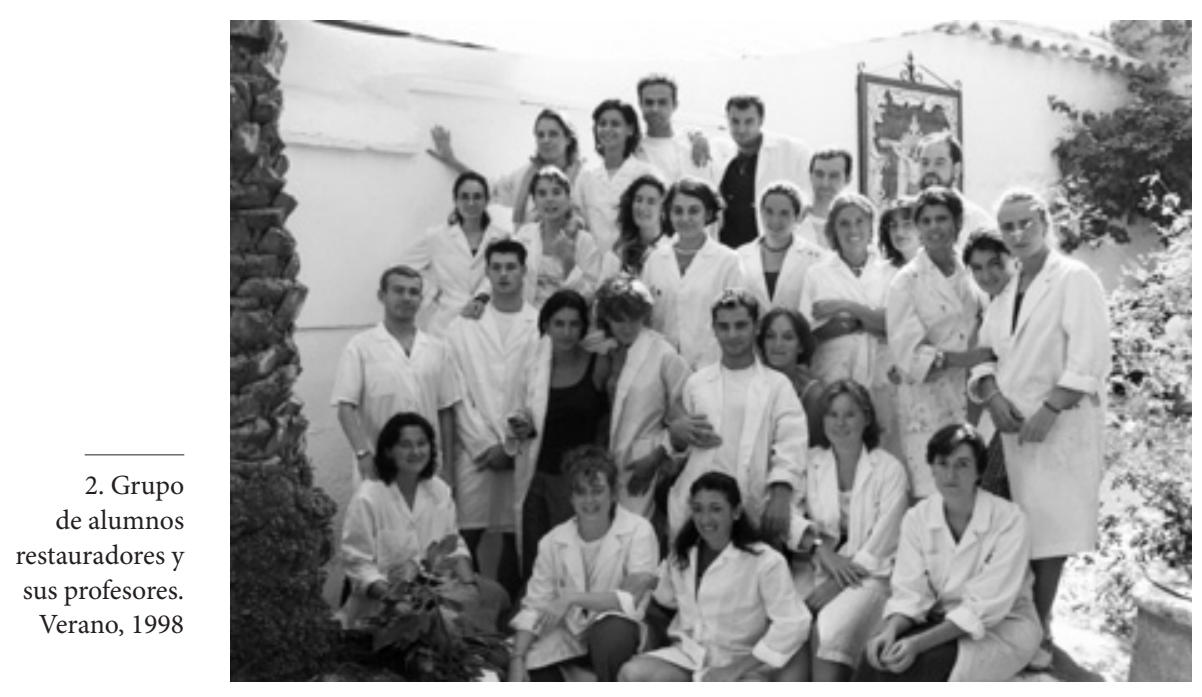

comenzó una labor restauradora que, en esos primeros momentos, llevaron a cabo las monjas clarisas de Mula. Este proceso era lento y los resultados no del todo satisfactorios. Por ello se buscaron otros cauces de intervención que agilizaran la actuación y garantizaran los mejores resultados.

\section{Los cursos de verano: de 1997 a 2001}

La idea de unos cursos de verano en que se procediese al estudio y restauración de la colección se gestó entre unos miembros directivos de la Asociación Cultural para la Defensa del Patrimonio, Siglo XXI, y el párroco de San Miguel, don Ramón. Se basaba en intentar reunir en Mula a alumnos de último curso de BeIlas Artes, especialidad de Restauración, de todas las universidades de España ${ }^{10}$ a fin de proceder con ellos a la restauración de las obras que así lo necesitasen, siempre dirigidos y supervisados por profesorado muy cualificado [2]. Con esta idea acudieron a quien esto escribe y entre todos se acabó de perfilar el formato. Los cursos durarían dos semanas en las que los alumnos estarían en régimen de internado que incluiría el hospedaje y la manutención completa. Para conseguir este requisito, absolutamente necesario por la procedencia dispar de los alum-

10 Para esos años aún no se había creado la especialidad en la universidad de Murcia, siendo las más cercanas y el lugar donde estudiaban los alumnos de Murcia, Granada y Valencia. 
nos que venían de todas partes de España e, incluso, del extranjero y que, además, facilitaba la relación alumno-profesor, así como la intervención sobre obra a destiempo y según lo requiriese el tratamiento aplicado, fue muy importante la colaboración municipal ${ }^{11}$ que cedió el uso de un albergue de juventud recién rehabilitado y que, por ello, reunía las mejores condiciones.

Pero como complemento de esa labor práctica que tanto iba a formar a los alumnos restauradores y tan beneficiosa sería para la colección, se propusieron unas conferencias teóricas que impartirían los más cualificados restauradores en activo en los más importantes museos, así como historiadores del arte que pudieran rellenar aspectos en los que los profesionales de la restauración estuvieran menos formados. Se elegirían especialistas en técnicas y soportes, e igualmente interesaban aquellos que hubieran tenido intervenciones recientes en obras emblemáticas o polémicas, como por ejemplo, El Caballero de la mano en el pecho o El Guernica ${ }^{12}$. Estas conferencias ocuparían las tardes y se abrirían a los alumnos de Historia del Arte y al público en general que estuviese interesado en saber y profundizar en estos importantes temas del arte. Así pues, el curso de verano se diseñó y realizó como teórico-práctico. Su nombre: Curso de Restauración, Conservación y Catalogación de Bienes Culturales. De él se realizaron cuatro ediciones, desde 1997 a 2001, dejando el año 2000 sin convocar y realizando dos turnos en el de 1997.

Ahora bien, no solo se trataba de restaurar las obras de la colección. Igualmente necesario era su estudio y catalogación y para ello contábamos con la dedicación de licenciados en Historia del que desde el principio estaban involucrados en el equipo.

\section{La actuación: procesos, equipo de trabajo y escenarios}

La empresa que nos proponíamos necesitaba de una buena organización y contar con un equipo de especialistas que se dedicase a cada uno de los menesteres ne-

\footnotetext{
11 Era entonces alcalde de Mula don José Iborra y concejala de Cultura, doña Elvira García Ballesta. También jugó importante papel don Manuel Ibernón Hernández, de agente de desarrollo local en Mula. 12 La restauración de la primera obra citada, llevada a cabo por Rafael Alonso, del Museo de Prado y especialista máximo en las obras de El Greco, levantó tal revuelo que nos parecía importantísimo que explicara los motivos que le llevaron a actuar de la forma que lo hizo. El restaurador aceptó gentilmente nuestra invitación y en este foro se expusieron y debatieron los criterios de restauración seguidos. En cuanto al Guernica, estábamos en medio de la polémica de si procedía su traslado o no, y Pilar Sedano, jefe de restauración del Reina Sofía, aclaró también su postura que se basaba sobre todo en el deterioro de la obra.
} 
cesarios para conseguir el buen fin. Ya se ha hablado del profesorado que se iba a dedicar a dirigir, orientar y supervisar el trabajo de los alumnos restauradores. Estos no debían pasar de los treinta y estaban en relación con el número de cuadros que se seleccionaba para intervenir y a su vez, de grado de deterioro, tamaño, dificultad de la técnica y soporte, etc. De tal forma que tampoco el número de obras solía pasar de las dos docenas. De esta forma, en las cuatro ediciones del curso se llegaron a restaurar un centenar de cuadros. La dirección técnica o, lo que es igual, las profesoras responsables fueron: Yolanda Closa Encinas, María Victoria Santiago Godos ${ }^{13}$ y María José Núñez Valentín, ayudadas por otros profesores, como lo fueron Juan Antonio Fernández Labaña ${ }^{14}$, Manuel Sánchez Rodríguez y Montserrat Rodrigo Zapata. Ya queda dicho que lo primero de nuestra labor era hacer la selección y una ficha técnica de cada obra en la que, además de los datos habituales, se apuntaba el estado de conservación soporte, capa de preparación, película pictórica), las necesidades de intervención y el proceso a seguir.

Al mismo tiempo se elaboraba la ficha de catalogación por los licenciados en Historia del Arte, Manuel Pérez Sánchez, Elena de la Ossa Jiménez y Josefina Jorquera Ramallo ${ }^{15}$. La coordinación y relación con las distintas entidades que colaboraban y patrocinaban corrió siempre a cargo de Juan García Sandoval ${ }^{16}$ y por fin, la dirección de todo corrió siempre a cargo de quien esto firma.

El coste total de esas dos semanas de intensivo trabajo era bastante elevado, ya que habíamos de correr con el alojamiento y manutención de los alumnos internos y equipo de profesores, así como contar con un apartado para dedicar al desplazamiento y modesto cachet de los profesores invitados que, como suele suceder en nuestra profesión (humanistas) los conseguíamos por amistad, más que por el "cebo" remunerativo. Para hacer frente a todo, en principio, solo contábamos con el importe de las matrículas de alumnos internos y externos. Lógicamente era algo más elevada la primera, pero aun así no cubría el total de gastos. Por ello fueron muy importantes las aportaciones que hicieron diversas entidades que creyeron en el proyecto y lo tomaron con la misma ilusión que los organizadores. Unos colaboraban con la cesión de locales, como el Ayuntamiento de Mula que dejó el recién renovado matadero que con buen acierto fue conver-

13 Actualmente la Dra. Santiago Godós es profesora titular y también vicedecana de la Facultad de Bellas Artes de esta universidad de Murcia.

14 Fernández Labaña es en el presente director del Área de Conservación del Centro de Restauración de la Región de Murcia.

15 El Dr. Pérez Sánchez es ahora profesor titular del Departamento de Historia del Arte. Las licenciadas, de la Ossa y Jorquera, profesoras de enseñanza media.

16 En la actualidad director del Museo de Bellas Artes de Murcia. MUBAM. 


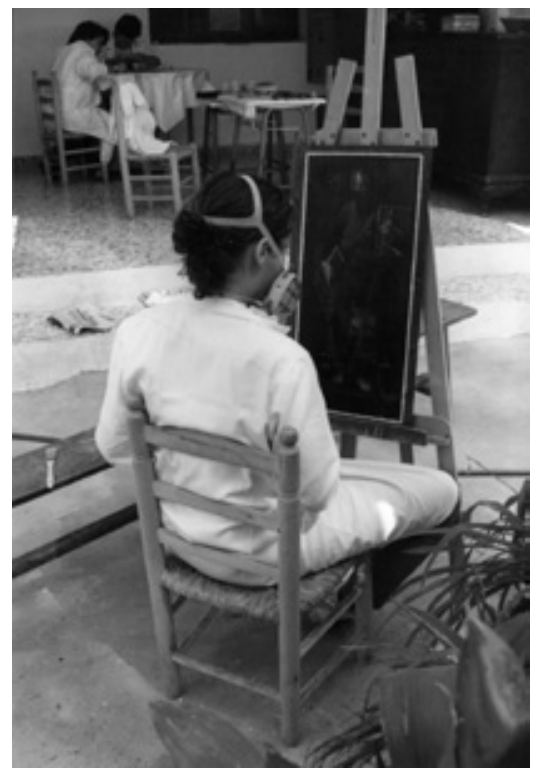

3. Proceso de limpieza con líquidos volátiles tóxicos

tido en albergue juvenil. O Caja Murcia que nos facilitaba el uso de su salón de actos en la oficina de la localidad, para allí impartir las clases teóricas. Aunque también fue muy importante la cesión del apartamento para investigadores con que contaba y cuenta el Museo de Arte Ibérico "El Cigarralejo», de Mula ${ }^{17}$, pues en él se alojaban los conferenciantes. E igualmente, decisiva fue la cesión de la planta terrena y patios que el párroco don Ramón nos hizo de la casa parroquial que él mismo habitaba en la planta superior. Con ello y dado que ahí estaba la mayor parte de la colección o si no, en el vecino templo, se facilitaba la actuación, sin tener que someter a los cuadros a traslados innecesarios.

Este local resultó idóneo para las distintas necesidades que conllevaban la catalogación y restauración. Contaba con cuarto de baño y cocina y por lo tanto, con agua corriente y focos de calor directo y además, al ser planta semienterrada, la morfología y distribución de sus habitaciones variaba tanto en condiciones climáticas, de luz y oscuridad, y hasta de seguridad que también favoreció los distintos procesos [3]. A todo se unía la posibilidad de usar los patios para la aplicación, en su caso, de sustancias tóxicas volátiles, por no hablar de los jazmines en flor, las buganvillas y otras delicias olorosas de los jardines mediterráneos. Las intervenciones restauradoras se hacían por las mañanas: desde las 9 a las 14 horas, pero no era raro ver restauradores en faena antes y después de esas horas por el compromiso que se llegaba a establecer en la obtención del mejor resultado. Igualmente, tras las clases vespertinas y la cena, se podían hacer comprobaciones de los procesos o retoques concretos que facilitarían la labor al día siguiente.

Como regla general se adoptó la de que todos los procesos de intervención fueran reversibles e igualmente, rehuir actuaciones muy drásticas, como la forra-

17 Fue esta, acción generosa y altruista de su directora que era y aún lo es, Dra. Virginia Page del Pozo. 


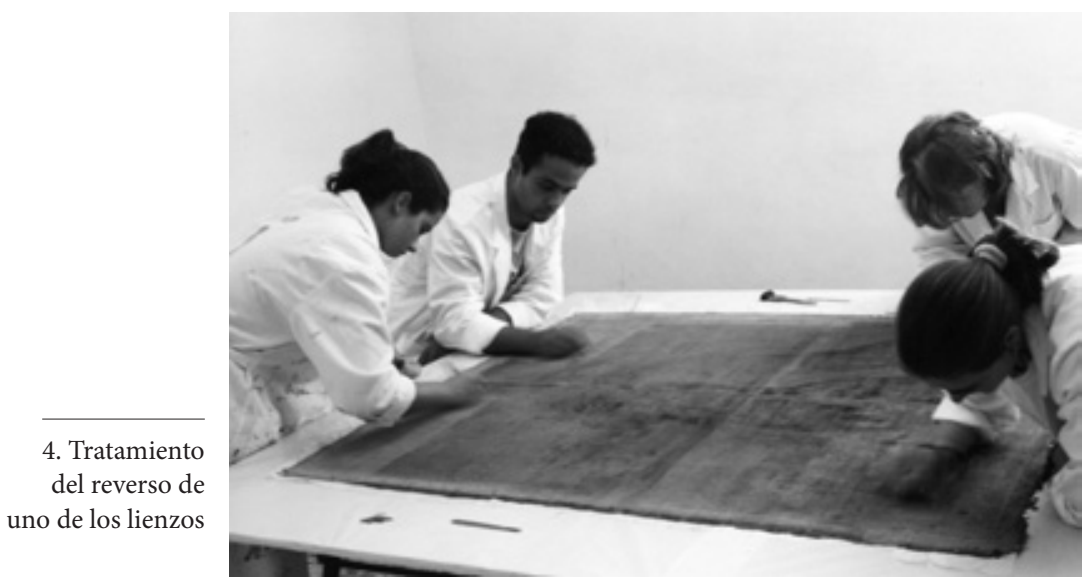

ción total, a no ser en caso completamente necesario [4]. Los barnices eran muchas veces y por su deterioro, levantados y sustituidos; los repintes eliminados; las carencias estucadas y la reintegración de pintura, siempre con acuarela y a punteado o rigatino (tratteggio). De todo se llevaba un minucioso seguimiento a fin de ir redactando desde el principio las detalladas y gruesas memorias finales que se entregaban a los organismos cooperantes y quedaron en copias guardadas por los directos responsables de la actuación.

Como ya hemos dicho, las clases teóricas se impartían por la tarde a razón de dos diarias y en ellas los alumnos de Restauración y los de Historia del Arte, internos y externos, tuvieron la posibilidad de escuchar a cualificado profesorado que exponía variados problemas y cómo resolverlos, aplicados a las obras que ellos, por su puesto de trabajo trataban todos los días. Vinieron a Mula, desde el Museo del Prado, también del Museo de Arte Reina Sofía, asimismo del Thyssen-Bornemisza, del Museo de Bellas Artes de Oviedo, de Patrimonio Nacional, de la Facultad de Bellas Artes de Madrid, del Consejo Superior de Investigaciones Científicas de Madrid, del Centro de Restauración de Murcia, y otros lugares especializados, no menos importantes pero que omito por no pormenorizar demasiado ${ }^{18}$. Entre otros temas, todos interesantes para expertos y profanos y que iban mucho más allá de lo que se llevaba entre manos, se habló de:

18 Pilar Sedano Espín, Reina Sofía; Ubaldo Sedano Espín, Thyssen; Rocío Dávila y María Teresa Dávila, Rafael Alonso Alonso, José de la Fuente Martínez, todos del Museo del Prado; Carlos Torreiro Luengo, Patrimonio Nacional; Consuelo Dalmau Moliner, Facultad de Bellas Artes, Universidad Complutense de Madrid; Clara González Fanjul, Museo de Bellas Artes de Oviedo; Rosa Rubio Pasamontes y Manuela Gómez, Museo Reina Sofía; María Luisa Tárraga Baldó, CSIC, entre otros. 
- La creación de un Departamento de Conservación y Restauración.

- La técnica del Greco vista por el restaurador.

- El problema de lo falso.

- La actuación sobre lo ya restaurado.

- La conservación preventiva en la Colección Thyssen.

- La problemática en la restauración del cartón y el papel.

- La restauración de esculturas en el siglo XVIII.

- Conservación de la escultura contemporánea: A nuevos problemas nuevas soluciones.

- El tratamiento de soportes en pintura sobre tabla.

- Tratamientos de conservación y restauración de materiales lapídeos.

- Desarrollo de la metalurgia y su restauración.

- La problemática de la firma en la pintura.

- Montaje y funciones de un Departamento de Conservación y Restauración en un Museo de Arte Contemporáneo.

- Técnicas de conservación y restauración del dorado.

- Pintura mural en la región de Murcia: Estado de conservación y restauración.

Además de otros asuntos más cercanos a la Historia del Arte, local o nacional.

Estas clases se desarrollaron desde el primer año en el Aula de Cultura de Caja Murcia, lugar con capacidad para cien alumnos, aforo que siempre se rebasaba con creces y había que completar con asientos movibles. Eran siempre clases muy activas y participativas que, al final, generaban intervenciones tan interesantes como la clase misma.

Pero considerábamos que tan importante era lo que se estaba haciendo, como darlo a conocer a la sociedad y no esperar para ello a la creación del Museo Parroquial que, desde el primer momento se deseaba y que al final, se consiguió, como más adelante veremos. De modo que, al final de cada curso se organizó una exposición con todo lo intervenido en esa edición, acompañado de paneles explicativos complementarios. El primer año se realizó en una sala de la Biblioteca de la localidad que reunía perfectas condiciones para ello. En otras ediciones se usó el Museo del Cigarralejo y salones de la misma parroquia de San Miguel. Lógicamente el acceso era gratuito y la afluencia de gente, numerosa.

Aunque no entremos en mucho detalle en el tema económico y puesto que ya hemos expuesto la cesión que de uso de locales nos hicieron: el Ayuntamiento, Caja Murcia y la parroquia de San Miguel Arcángel, si citaremos aquí 
otro organismo que nos fue de trascendental importancia, Iniciativa Leader II, que colaboró en las cuatro ediciones con cantidad suficiente para que, junto a los ingresos por matricula, poder abordar dignamente la manutención del alumnado interno y el pago de profesorado. Junto a ello, Siglo XXI, facilitaba toda la infraestructura y de nuevo la parroquia de San Miguel corrió con el gasto de materiales, si bien se utilizaron también algunos aparatos más específicos que el propio profesorado aportaba para uso general. Por último, toda la gestión administrativa pasaba por la Universidad de Murcia, ya que los cursos fueron editados como Cursos Extraordinarios de la Universidad del Mar.

\section{Las pinturas: temas y soportes}

La colección es rica en número de obras y temas así como en soportes y técnicas, abarcando, además, un amplio periodo de tiempo en su cronología que se extiende desde finales del siglo XVI al primer tercio del XX. Ronda la cantidad de las doscientas piezas, entre cuadros y otros objetos, incluyendo aquí las propias pinturas de doña Pilar de la Canal Rosique, la donante, pintora ella misma, que resultan más interesantes por ser demostración de las aficiones y gustos altoburgueses del momento que por su propio valor artístico. Lamentablemente no se encuentra el primer inventario que debió de hacerse en el momento de la donación. Cuando se hizo la citada exposición del año 1987 se contaron 131 obras, pero en otro inventario mecanografiado, sin fecha ni firma de autor, son 169 las registradas ${ }^{19}$.

Como se viene diciendo, las obras de mayor envergadura y temática religiosa colgaban de los muros de la iglesia parroquial. Pero otras muchas de esta temática y asunto profano, se custodiaban en otras dependencias de la parroquia ya que, si bien se adecuó un primer y rudimentario "museo" al poco de su donación, con el paso del tiempo este se fue abandonando y las obras se recogieron y custodiaron de la mejor manera posible por los distintos responsables que iban haciéndose cargo de la parroquia. No impidió esto su deterioro y así se hallaban cuando nosotros comenzamos el estudio, catalogación y restauración en aquel el verano de 1997 y finalizó en el de 2001, dando como resultado el montaje de un museo, más capaz y mejor adecuado que el primero, en el que

19 Archivo de la parroquia de San Miguel de Mula, carpeta correspondiente a los documentos de la colección. 
ahora se exhiben ${ }^{20}$, a la espera de que puedan realizarse las necesarias mejoras de infraestructura para la perfecta conservación de la colección.

Hay varias obras firmadas por notables artistas locales, como Joaquín Campos, siglo XVIII o Antonio Meseguer, siglos XIX-XX. Igualmente las hay también fácilmente atribuibles a otros pintores del territorio, como Camacho Felices, lorquino del siglo XVII-XVIII. También se han encontrado firmas de otros importantes pintores de ámbito nacional como lo son: Antonio María Esquivel, primera mitad del siglo XIX y Francisco Preciado de la Vega, segunda mitad del siglo XVIII21. Asimismo hay obras que pueden atribuirse con bastante seguridad a algunos pintores de importancia como son el bodegón con gallos, del círculo de Tomás Yepes, o Hiepes, valenciano, del siglo XVII. O, por citar alguna de las más hermosas obras, la Cabeza de filósofo anciano que acusa la perfección e iconografía de Antonio Rafael Mengs. Algunas obras anónimas son sobresalientes, como sucede con la Santa Águeda de escuela napolitana, la Batalla de Aníbal o los paisajes apaisados de campo y mar. El valor artístico en conjunto es notable, pero asimismo la colección adquiere un interés incuestionable por la variedad temática, la abundancia de pintura sobre cobre y las muchas muestras de pintura sobre cristal.

Entre la pintura religiosa se encuentran los temas más habituales de la vida de Cristo: infancia y pasión, y son abundantes las centradas en el Nacimiento con cuatro representaciones de la Adoración de los Pastores y dos de la Epifanía, así como una Huida a Egipto, popular de sabor americano, de singular iconografía. Asimismo son bastantes los cuadros que recogen a María en variadas advocaciones, siendo cuatro las representaciones como Inmaculada (una de ellas, solo la cabeza que parece querer copiar la del convento de san Francisco, de Salzillo y está firmada por Joaquín Campos en 1786), cinco como Madre con el Niño en brazos. Una Virgen del Carmen con ánimas, también de Joaquín Campos puede ser el boceto para el bocaporte de la capilla del Carmen, de Mula. Y una interesantísima copia de la Dolorosa de Salzillo, aún del siglo XVIII, anónima, que nos informa de la forma que iba vestida en los primeros momentos, según consejo del mismo escultor. Para cerrar, aunque no agotemos este tema, señalaremos una curiosa versión de Santa María de Ocotlan, venerada en la ciudad de Tlaxca-

20 Que, tras el traslado a otra parroquia de don Ramón García y por desidia y abandono de los responsables de la parroquia que le han sucedido, desgraciadamente, también va en proceso de degradación, afectando con ello las obras que con tanto mimo y celo se restauraron en su momento.

21 RAMALLO ASENSIO, G., "Aportaciones a la obra pictórica de Francisco Preciado de la Vega, pintor sevillano en Roma", Laboratorio de Arte. Homenaje al profesor Juan Miguel Serrera, 12, Sevilla, 1999, pp. 293-300. 


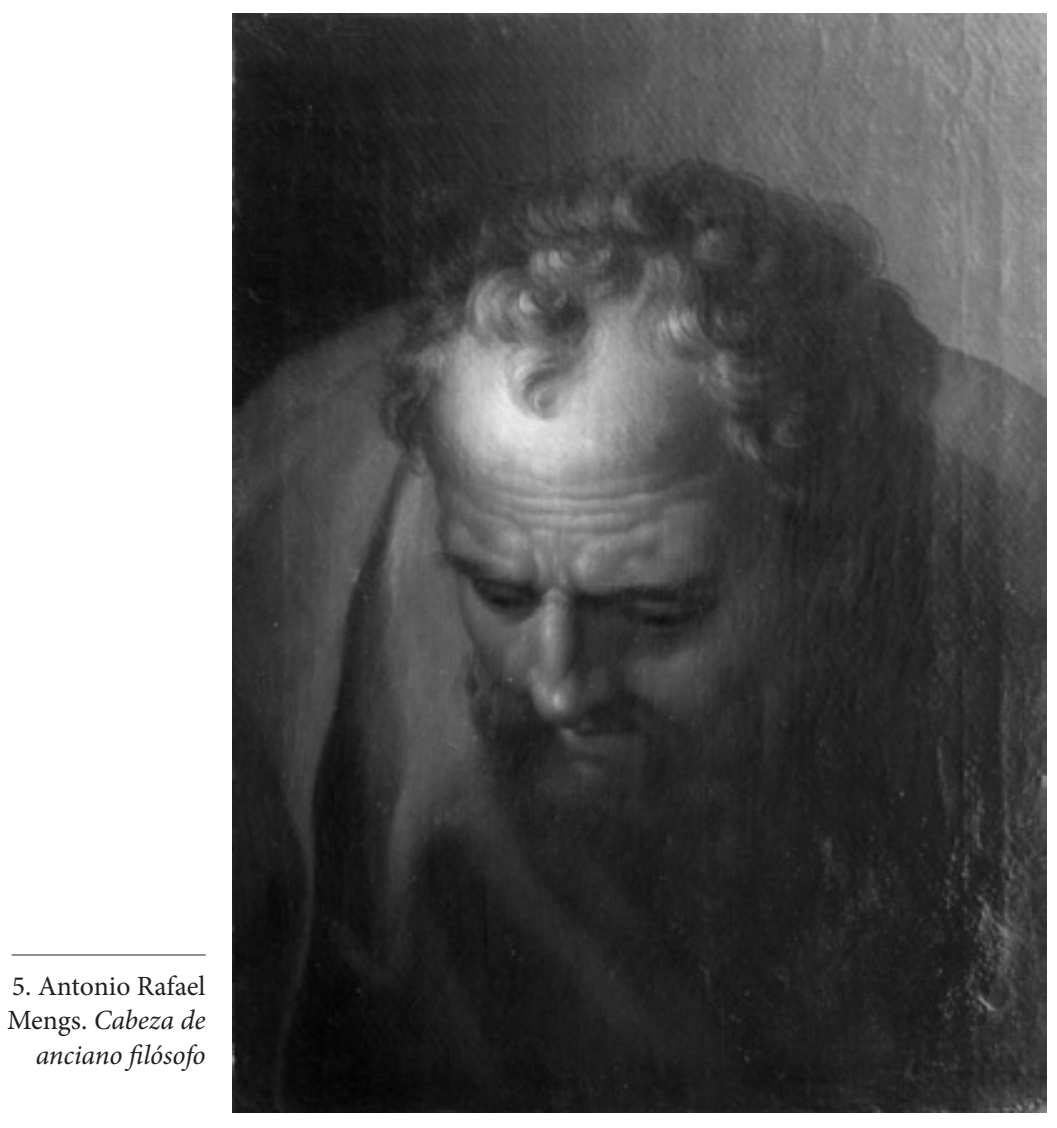

la, cerca de Puebla de los Ángeles, firmada y fechada, y con inscripción del siglo XVIII que autentifica su procedencia.

Esta última pieza da noticia de los "recuerdos piadosos" de posibles viajes que hubiera realizado algún miembro de los que enriquecieron la colección y estos pueden explicar la existencia de pequeños cobres, claramente de devoción, como lo es uno que representa a san Antonio de Padua que por el reverso está fechado en 1739 y acoge la siguiente inscripción: "Vera efigies divis Antonÿ Patavini ab originale ad vivum desumpta 18-July-1738".

Es de indudable calidad e importancia la noble Cabeza de anciano [5] que tras el estudio y restauración últimos se ha podido poner en paralelo con la figura de San José que pintó Antonio Rafael Mengs (Aussig, Bohemia, 1728-Roma, 1779) en 1765, en la Sagrada Familia destinada a los reyes de España Carlos III y 
María Amalia de Sajonia, que ahora se encuentra en la Colección Wellington de Londres. En un principio llegué a pensar en que fuera un modelo presentado a los reyes, pero tras más profundo estudio lo creo anterior y quizás hecho como ensayo de claroscuro en Italia y, más tarde, usado como modelo para san José en el citado cuadro ${ }^{22}$. Hay también dos apostolados, uno en lienzo (círculo valenciano del primer tercio del siglo XVII) y otro sobre cristal, obras del XVII y XVIII, respectivamente que están figurados en tamaño de busto y asimismo, también sobre cristal, los cuatro evangelistas. $Y$ en cuanto a los santos hay muestras que van desde los de representación más frecuente, como San Pedro y San Pablo (dos bonitas tablas alargadas, de 64×33' $5 \mathrm{~cm}$, toledanas de finales del XVI, quizás círculo de Luis de Velasco (+1606), San Juan Bautista, San Francisco, San Antonio, Santo Tomás (gran lienzo de $188 \times 115 \mathrm{~cm}$ ), Santa Apolonia (del siglo XVII y estética clasicista napolitana) o Santa Teresa, a otros de devoción mas ocasional, como San Pelegrín, o devoción muy local como es el caso de San Ildefonso en el momento de la imposición de la casulla, de quien hay dos buenos cobres de distintos tamaños. Pero cobra una especial importancia la representación de Sor María Angela Astorch, lienzo en estricta relación con la historia local que sin embargo aparece pintado en Roma por Preciado de la Vega en 1773, según se descubrió en el proceso de restauración en el verano del $98^{23}$.

Pero si importante es el grupo de pintura religiosa, no lo es menos el de pintura profana y nos informa de los gustos refinados del coleccionista. Son destacables dos lienzos muy apaisados $(48 \times 162 \mathrm{~cm}$ ) que recogen una Escena Naval y un Paisaje de campo que ya han sido atribuidos a Juan de Toledo ${ }^{24}$, tipo de pintura muy frecuente en las casas del XVII y XVIII, si bien muy escasos en la actualidad, y también tiene interés la Batalla de Aníbal $\left(108 \times 165^{\prime} 3 \mathrm{~cm}\right)$, de la segunda mitad del XVII.

Asimismo hay varios bodegones de diferente calidad; los mejores, realmente excepcionales, son dos de pequeño formato: uno Bodegón de los higos (34 x

22 RAMALLO ASENSIO, G., "Antonio Rafael Mengs, posible autor de una Cabeza de filósofo anciano, de la Colección La Canal-Blaya. Museo de San Miguel Arcángel de Mula, Murcia», In Sapientia Libertas. Escritos en homenaje al profesor Alfonso E. Pérez Sánchez, Madrid-Sevilla, Museo Nacional del Prado/ Fundación Focus-Abengoa, 2007, pp. 587-593.

23 Véase nota 21. Desde el descubrimiento de su firma el cuadro ha sido expuesto en dos ocasiones: una de ellas en la Sala de Exposiciones de Las Claras (comisario, Cristóbal Belda Navarro) que generó el catálogo: Paraísos perdidos. Patios y clausuras, Octubre-Noviembre, 1999, p. 288-289 y la otra en Huellas, Catedral de Murcia, 2002, p. 487. La firma da fe de autor fecha y lugar: FRANcus PRECIADO Fe ROMA An 1773. Y por la inscripción se da cuenta de la figura representada y sus virtudes: LA Ve. Me. MARÍA ÁNGELA ASTORCH FUNDADORA DEL CONVENTO. DE CAPUCHINAS DE LA ENCARNACION DEL Sso. Sa/CRAMENTO.

24 IBORRA BOTIA y MUÑOZ CLARES, Pinturas de los siglos XVI al XIX..., sin numerar. 


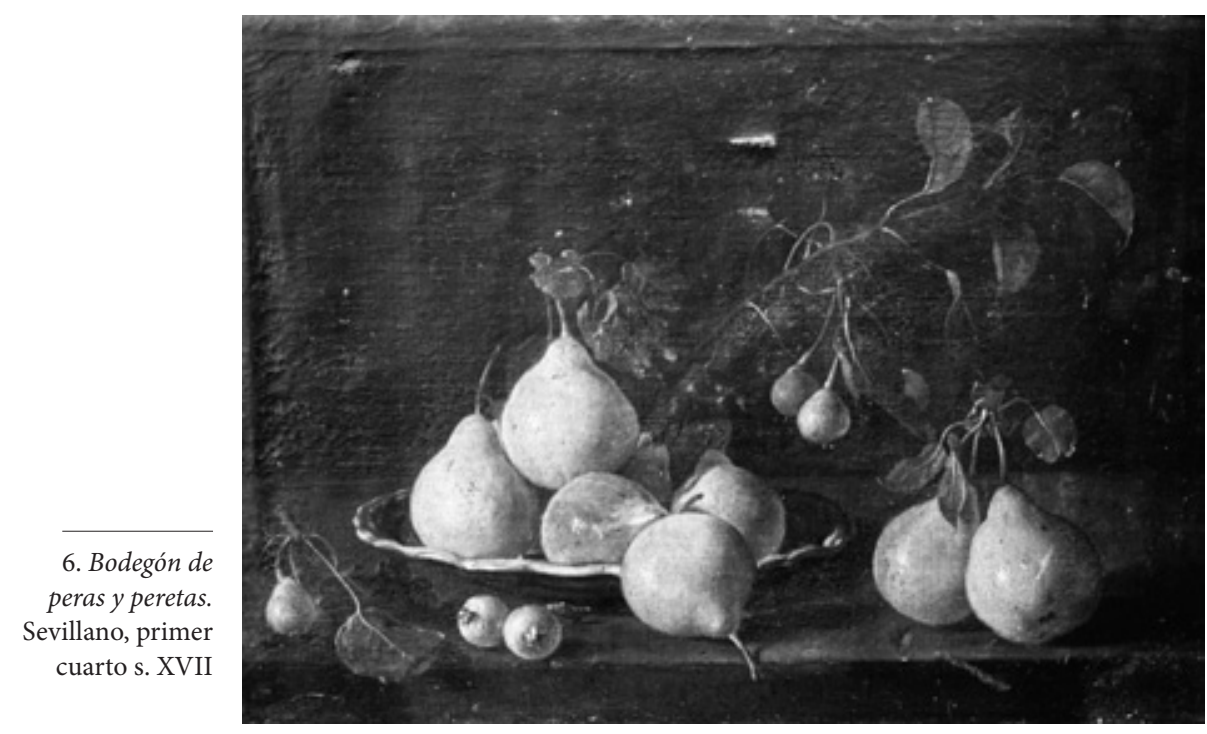

47 '5 cm) y el otro de iguales medidas, Bodegón de las peras y peretas [6], quizás sevillanos, del primer cuarto del siglo XVII; el primero presentaba un roto considerable en el centro de la superficie pintada que fue subsanado con injerto y parche (siempre se procuraba evitar el reentelado) y el segundo, por su buen estado de conservación y su posibilidad de importante autoría, se dejó sin intervenir, a la espera de poder hacerlo en otro contexto, por personal muy especializado y paralelo a estudio riguroso ${ }^{25}$. Otro que también presenta buena calidad e interés indudable, de mayor tamaño $\left(104 \times 155^{\prime} 5 \mathrm{~cm}\right)$ y buena conservación general, representa Flores con gallos y puede ser atribuido casi con seguridad al valenciano Tomás Yepes (+1674), especialista en estos bodegones de colores muy vistosos y composición preciosista ${ }^{26}$. También otros dos bodegones restaurados en la campaña del 98 representan la carne y el pescado: Carnestolendas y Cuaresma, el segundo muestra una calidad muy superior al primero, que incluso parece posterior y con añadidos para hacerlo de tamaño semejante al otro $\left(38^{\prime} 5 \times 58 \mathrm{~cm}\right)$.

25 En el verano de 2001 tuvo lugar el último curso de restauración por haber concluido las ayudas y subvenciones. A partir de entonces el cuadro quedó custodiado en la parroquia y no expuesto en su museo, con lo cual se retrasaba su restauración y estudio. Quien esto escribe perdió toda relación con tan importante asunto. Si bien el cuadro se ha restaurado el pasado año en un taller local, con unos resultados más que cuestionables y ha quedado expuesto en una de las salas del museo.

26 Esta atribución se debe a información oral de don Alfonso E. Pérez Sánchez que visitó los cursos un par de años, interesado vivamente por la colección y lo que se estaba haciendo. Agradezco su generosidad intelectual. 
Pero aún existe otro grupo dentro de la pintura profana que nos parece del máximo interés y no tanto por su cualidad artística, que la tiene, sino por la relativa rareza de su contenido. Se trata de cuatro cuadros de pequeño tamaño ( $44^{\prime} 5$ x $32 \mathrm{~cm}$ ) que representan a cada uno de los cuatro continentes: Europa, Asia, América y África, y dos ovalados $(32 \times 28 \mathrm{~cm}$ ) con sendas alegorías del Verano y el Otoño. Y por último, son también del máximo interés por su rareza, once Signos del Zodiaco (falta Géminis), de $27 \times 20 \mathrm{~cm}$. Todos ellos pueden ubicarse en la segunda mitad del siglo XVIII: aún barrocos, aunque con elementos rococó los continentes, más decididamente rococó las estaciones y con clara estética neoclásica, casi imperio, los once cuadros que componen ${ }^{27}$ el Zodiaco. Todos están figurados en bellas mujeres que pasan de la gracia a la gravedad, pero así como los dos primeros grupos pueden relacionarse con la pintura levantina, el último, de extremada rareza, nos pondría en contacto con el mismo París revolucionario. $Y$ ya para terminar, señalaremos los retratos, que también los hay, y uno muy bueno, Retrato de un militar, firmado en 1836 por A. Esquivel (Antonio María Esquivel y Suárez de Urbina, Sevilla, 1805-Madrid, 1857).

La técnica más empleada es el óleo aplicado a los distintos soportes de: lienzo, tabla, cobre, latón y cristal, pero también hay unos bonitos ejemplos de pastel sobre papel en tres paisajes firmados por el pintor murciano Francisco Meseguer en $1913^{28}$ (15). Y como decimos los soportes son muy variados. Desde luego predomina el lienzo que alcanza dimensiones considerables en algunas obras: Sto. Tomás de Aquino en Gloria o, Dolorosa copia de Salzillo, o la misma Visión de Sor María Astorch, así como en la Marina y Paisaje atribuidos a Juan de Toledo, la Batalla de Aníbal, y el bodegón del círculo de Yepes. Pero lo más habitual es el tamaño mediano o pequeño, propio de cuadros de devoción para los hogares de la clase adinerada.

Llama la atención la gran abundancia de cobres y estos de variada procedencia; los hay flamencos, también italianos y españoles, e incluso alguno que puede situarse como obra centroeuropea [7]. De entre ellos los hay de tamaño mediano: una Imposición de casulla a San Ildefonso (53 × 42 ‘ $5 \mathrm{~cm}$ ) o una preciosa Última Cena (34 × $26 \mathrm{~cm}$ ), aunque lo más normal es el tamaño pequeño como la otra Imposición de casulla a San Ildefonso (12'5 x $10 \mathrm{~cm})$, una Virgen en

27 Hacemos esta afirmación por haber localizado un grabado de Louis Lafitte que representa la lluvia y que ilustraría un calendario republicano, del cual es copia exacta el cuadrito que representa a Acuario. 28 De este pintor hay otros dos cuadros más, un retrato y un ramo de flores, además de dos copias de Velázquez (cabeza de Felipe IV y la cabeza del Niño de Vallecas) que le pueden ser atribuidos; ello da que pensar en una amistad con la familia Saavedra o Blaya e informa del enriquecimiento de la colección aun en tiempos recientes. 


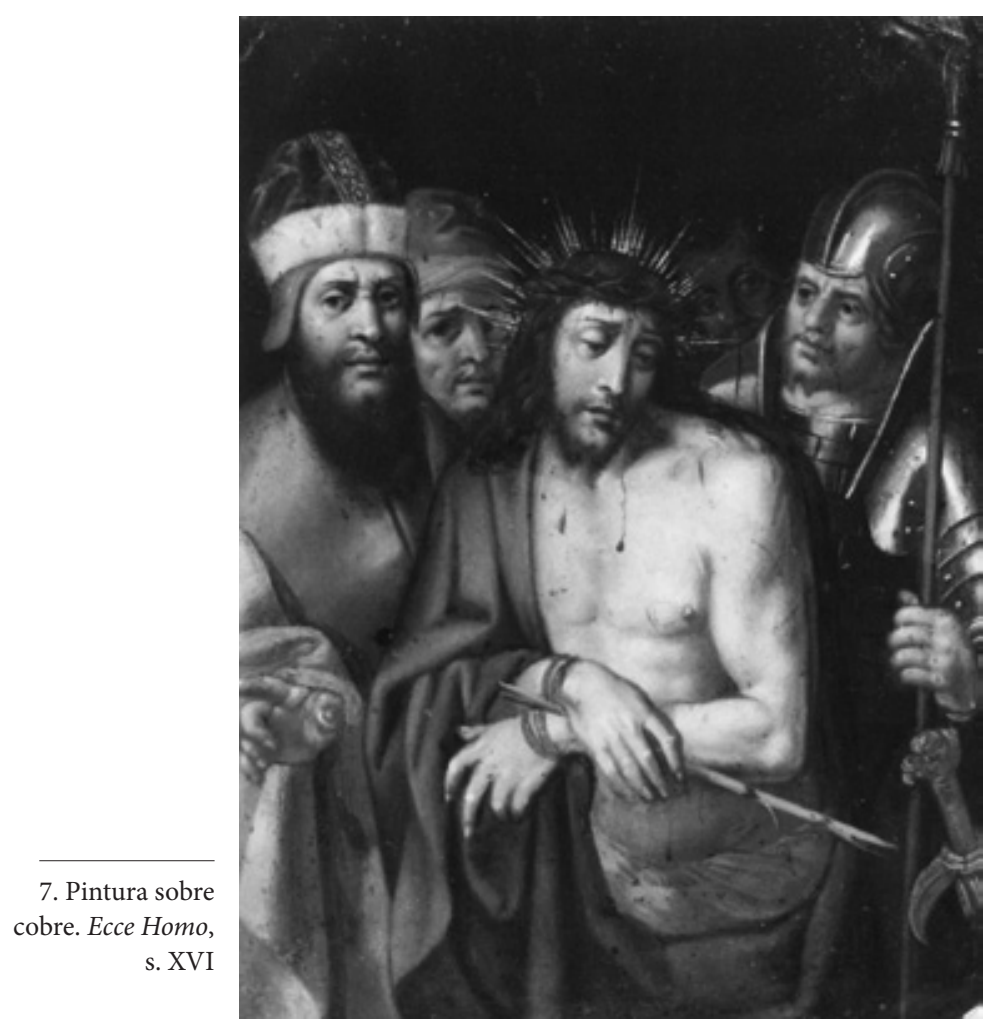

meditación (12'8 x 9'8 cm), o un Crucificado con la Magdalena (23'5 x $18 \mathrm{~cm}$ ). Hay también un ejemplo de óleo sobre latón $\left(25^{\prime} 5 \times 17^{\prime} 8 \mathrm{~cm}\right)$ que es importante por su calidad y tema: Una cita amorosa. También hay buenos ejemplos de óleo sobre tabla como los ya citados San Pedro y San Pablo, toledanos del último tercio del siglo XVI, un Cristo Eucarístico de buena factura y principios del XVII, o un San Juan Bautista que puede ser una notable copia del XVII, de algún original de Amberes de finales del siglo XVI, cuya madera de soporte, según el experto del Museo del Prado, José La Fuente, en calidad y técnica de ensamblaje, podrían ser flamencos.

Pero lo más notorio es la gran abundancia de obras pintadas al óleo sobre cristal, técnica muy de moda en el siglo XVIII ya avanzado que exigía una gran maestría en el pintor por no admitir el retoque, pero que ofrecía un resultado final muy vistoso, lleno de color y luz (en los mejores casos próximo al efecto del pastel) y no resultaban caros para el coleccionista. De estos hay todo un apostolado, doce 


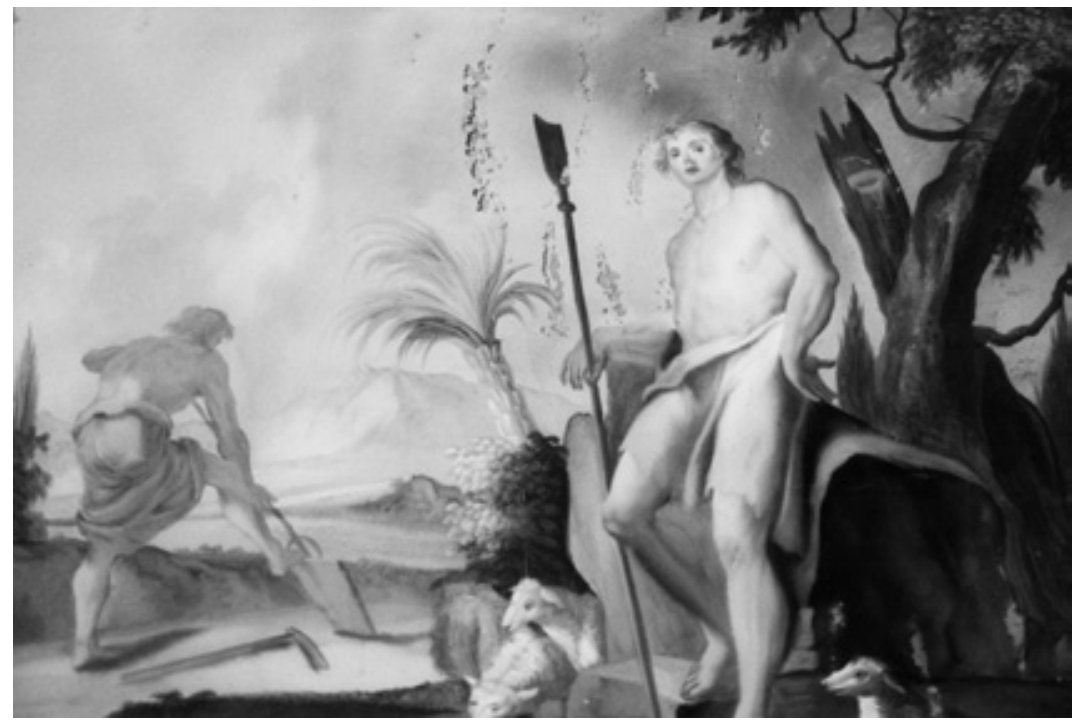

8. Pintura sobre cristal. Historia de Caín y Abel. Abel honra a Yahvé. Últimas décadas del siglo XVIII

cuadros $^{29}$; una serie de los cuatro evangelistas y otra, curiosísima por el tema, que está compuesta de cuatro cuadros con escenas de la vida de Caín y Abel: Caín y Abel como agricultor y pastor [8], Las ofrendas a Dios, El crimen de Caín y por último, La reprensión de Dios. Por supuesto existen buenos grabados, algunos coloreados (los que representan a San Isidoro, San Fulgencio y Santa Florentina) y un precioso bordado de buen tamaño que representa a Cristo Eucarístico.

\section{El Museo}

Todos los esfuerzos que se realizaron en ese periodo de tiempo y con los cuatro cursos intensivos de catalogación y restauración, tenían como fin que las obras se pudieran custodiar, conservar y exhibir al público en un ámbito adecuado. Desde luego la parroquia que era la poseedora de los bienes resultaba el lugar

29 En realidad, aunque cada uno de los apóstoles vaya identificado con el instrumento que define su particular iconografía, parece que el anónimo pintor hubiera querido plasmar ante todo una impresionante galería de cabezas de distinta edad y tipo facial, que además adoptan posturas forzadas y muy movidas o vistas desde ángulos inusuales. Ante ello recordamos los grabados de Domenico Tiépolo reunidos en el libro: Raccolta di teste, 1770. 
9. Museo de San Miguel Arcángel. Mula. Planta

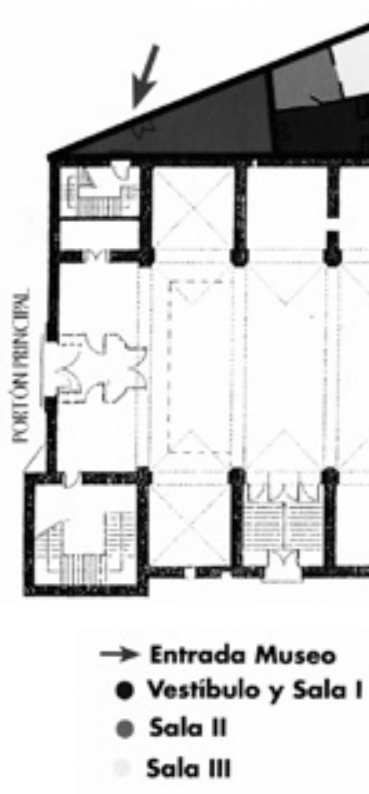

- Sala IV

- Sala V

- Patio

más adecuado y no hubo posibilidad de acuerdo entre su representante y las autoridades municipales que ofrecían otras sedes. Por ello, durante el año 2000, último en que se harían actividades, se llevaron a cabo obras de ampliación y adecentamiento de espacios anexos al templo: sacristías, salones parroquiales y otros de nueva construcción, a fin de que allí se montara ese nuevo museo en el que las pinturas restauradas y las que no estaban muy deterioradas, pudieran ser admiradas por la sociedad a la que le fueron legadas.

Es cierto que no se podían conseguir los grandes espacios diáfanos e iluminados con luz natural que ahora diseñan los arquitectos estrella. Todo era pequeño, en distintos planos y malas entradas de luz [9]. Pero allí cabían los aproximadamente cien cuadros que se habían recuperado y estudiado y lo que es mejor, se había cuidado la seguridad de todas las salas. La iluminación artificial tampoco era la más adecuada para favorecer los cuadros: luz blanca de neón, pero si era suficiente al menos en las salas más oscuras.

Al final se habían conseguido cinco salas: cuatro anejas al lado norte de la iglesia y de entre ellas, una más grande era la sacristía del siglo XVIII. Aún quedaban otras dos sacristías más pequeñas a uno y otro lado del presbiterio; 


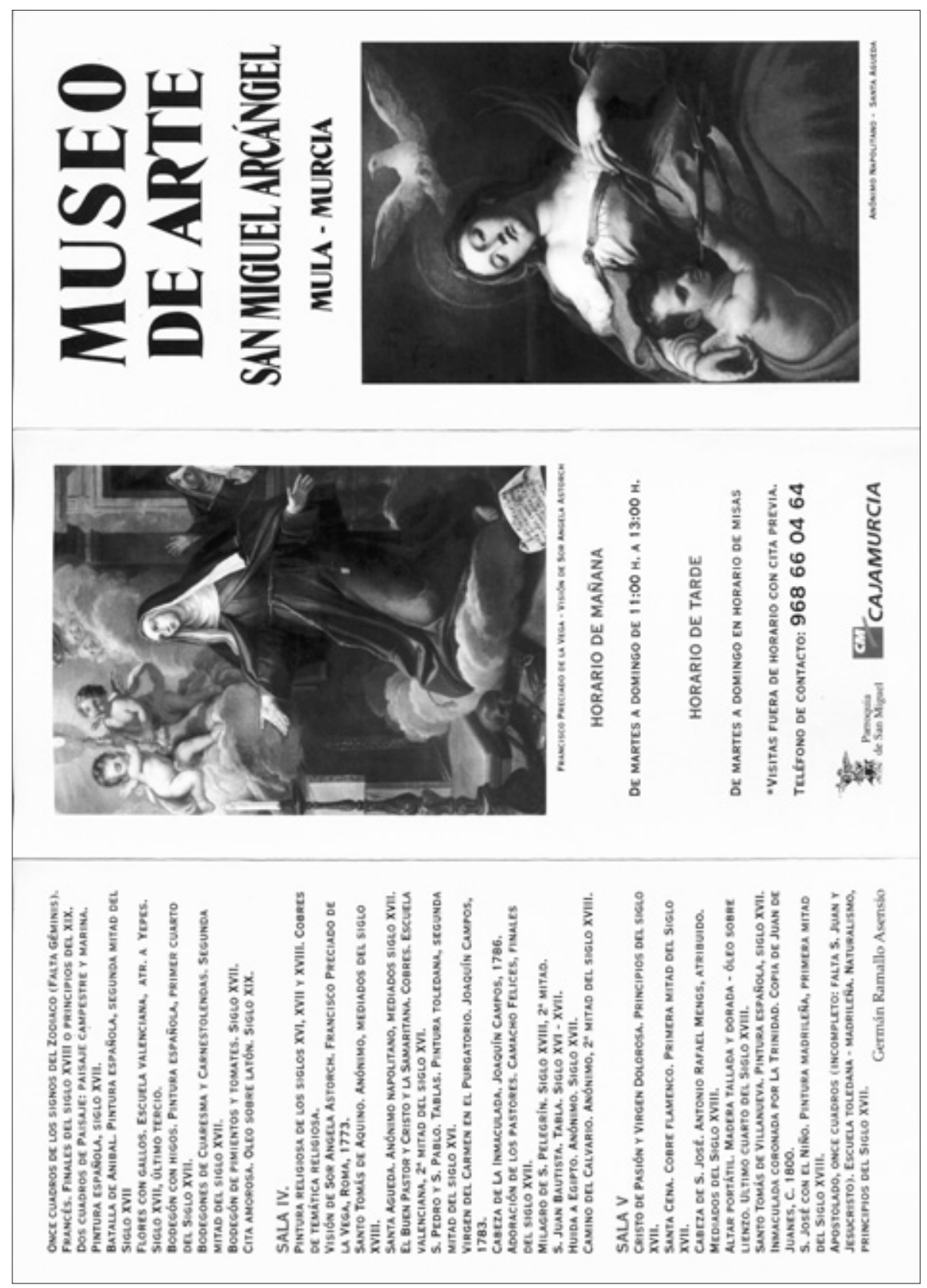

10. Museo de San Miguel Arcángel. Mula. Tríptico 
la de su izquierda quedó para hacer las funciones de ello y la de la derecha, la pieza más antigua del templo, de la primera mitad del siglo XVI, también pasó a formar parte del museo y en ella por su ubicación más recóndita y su seguridad desde todos los flancos, se colocaron las mejores obras. $Y$ con esto llegamos a otro tema importante: la distribución de obra. Esto fue algo muy meditado, pero hubo de hacerse en función de los condicionantes del espacio. Desde luego los grandes lienzos de tema religioso quedarían en las paredes del templo, tal y como habían estado desde la donación, aunque alguno que así lo estaba antes de la restauración se decidió exponer debido a su interés artístico. En cuanto a lo demás, se eligió la distribución temática: lo profano (retratos, paisajes, bodegones, cuatro continentes, estaciones y zodiaco, iría a las salas dos y tres). El vestíbulo se concibió en dos zonas, la delantera acogió los cuadros de doña Pilar de la Canal y algunos otros que tenía que ver con la colección o el espíritu de fin de siglo XIX que había generado la donación, y la zona trasera, a nivel más elevado y semicerrada por barandilla de madera acogió las numerosas y frágiles pinturas sobre cristal. La pintura de tema religioso de pequeño y mediano tamaño, incluidos los numerosos cobres, se colgó en la sacristía grande, que tiene mucha superficie de pared pues solo se ilumina con ventana al fondo y está situada muy alta. Para terminar con lo religioso, de pequeño o mediano tamaño y más valioso en calidad artística que, como ya he dicho, ocupó la sacristía antigua; también con única ventana, igualmente abierta en zona muy alta del muro sur.

La inauguración se hizo al finalizar el último curso: en agosto de 2001 con asistencia del alcalde de Mula, don José Iborra, el rector de la Universidad, don José Ballesta y el obispo de la diócesis, don Manuel Ureña Pastor. Para el acontecimiento se preparó un tríptico informativo que se sigue dando a los visitantes actuales y se escribieron textos murales, explicativos de cada sala y las obras que contenía [10].

Ese "final feliz" duró lo que el párroco que se había comprometido tanto en la labor. Cuando este fue trasladado a otra localidad, el museo fue languideciendo y a ello también ayudó el hecho de que ni las autoridades municipales, ni las autonómicas, al no tener la propiedad, lo incluyeran en campañas divulgativas de la ciudad: una vez más la rentabilidad política se antepuso al beneficio puramente cultural. 
